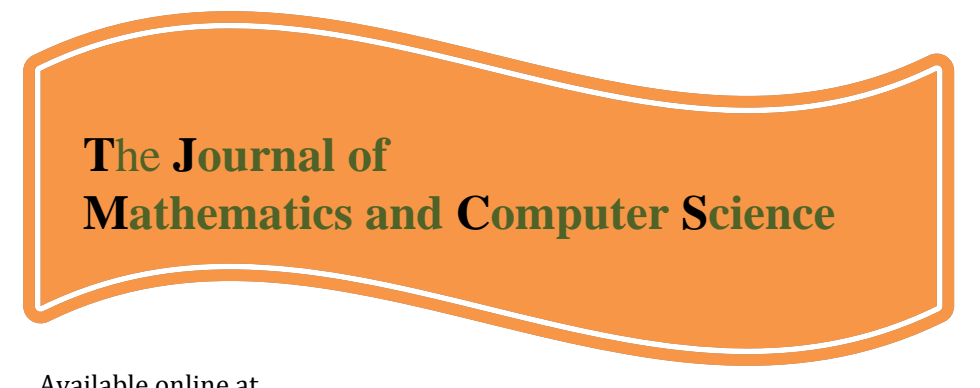

http://www.TIMCS.com

The Journal of Mathematics and Computer Science Vol .2 No.1 (2011) 1-8

\title{
Fuzzy Relational Dynamic System with Smooth Fuzzy Composition
}

\author{
Arya Aghili Ashtiani1,*, Mohammad Bagher Menhaj ${ }^{2}$ \\ Amirkabir University of Technology, arya.aghili@aut.ac.ir \\ Amirkabir University of Technology,mbmenhaj@yahoo.com
}

Received: September 2010, Revised: November 2010

Online Publication: January 2011

\begin{abstract}
Fuzzy relational models of functions have been developed in recent two decades which has led to fuzzy relational models of dynamic systems which we call fuzzy relational dynamic systems (FRDS). In this paper the effectiveness of smooth fuzzy relational compositions (FRC) in such dynamic models is studied after introducing a general framework for modeling of dynamic systems using FRDS, and so the smooth FRDS is developed. A modeling structure is presented in this regard as well as a related identification algorithm. Finally, the modeling capability of the proposed smooth FRDS is verified via some simulations on various benchmark problems and actual dynamic systems.
\end{abstract}

Keywords: Fuzzy relational dynamic system, smooth fuzzy relational composition, fuzzy relational modeling.

\section{Introduction}

A fuzzy relational dynamic system (FRDS) is a fuzzy relational model (FRM) which models a dynamic system based on the function approximation. An FRM is based on the fuzzy relational equations (FREs) introduced in 1976 by Sanchez in [1]. A typical FRE is written as $\underline{b}=\underline{a} \circ R$, in which $\underline{a}$ and $\underline{b}$ are fuzzy vectors and $R$ is a fuzzy relational matrix. The elements of $\underline{a}, \underline{b}$, and $R$ all belong to

\footnotetext{
${ }^{1,{ }^{*}}$ Corresponding author: PhD student at electrical engineering department, working on advanced intelligent and hybrid systems.

${ }^{2}$ Professor at electrical engineering department working on advanced control systems including advanced intelligent systems.
} 
$[0,1]$. The fuzzy relational composition "o" is of type s- $t$ in almost all applied papers while it can also be of type t-s from the theoretical approach, where " $\mathrm{t}$ " and " $\mathrm{s}$ " stands for t-norm and t-conorm respectively. This equation is evaluated as $b_{j}=s_{i=1}^{q} t\left(a_{i}, r_{i j}\right)$. The equation may model a static function $f$, which can be used to model a dynamic system, as shown in Fig. 1. This leads to the socalled FRDS.

FRDS is in fact an extension of fuzzy linguistic systems in which each rule has a truth value between zero and one. Many papers are available about function approximation and model identification by FRMs, such as [2]-[4], but most of them are based on nonsmooth fuzzy relational compositions and some of them have used some specific types of smooth fuzzy relational compositions, such as sum-prod fuzzy relational composition in which the t-norm is the algebraic product and the t-conorm is the algebraic sum. In this paper we present a general framework for FRDS based on any smooth fuzzy relational composition and we will show that using smooth fuzzy relational compositions improves the modeling capability of the FRDS. This work generalizes the work done in [5] for the static case.

Sections 2 and 3 represent respectively the two important parts of the modeling process, the modeling framework and the identification algorithm. The results are verified for several benchmark problems in Section 4. A brief conclusion in Section 5 wraps up the paper.

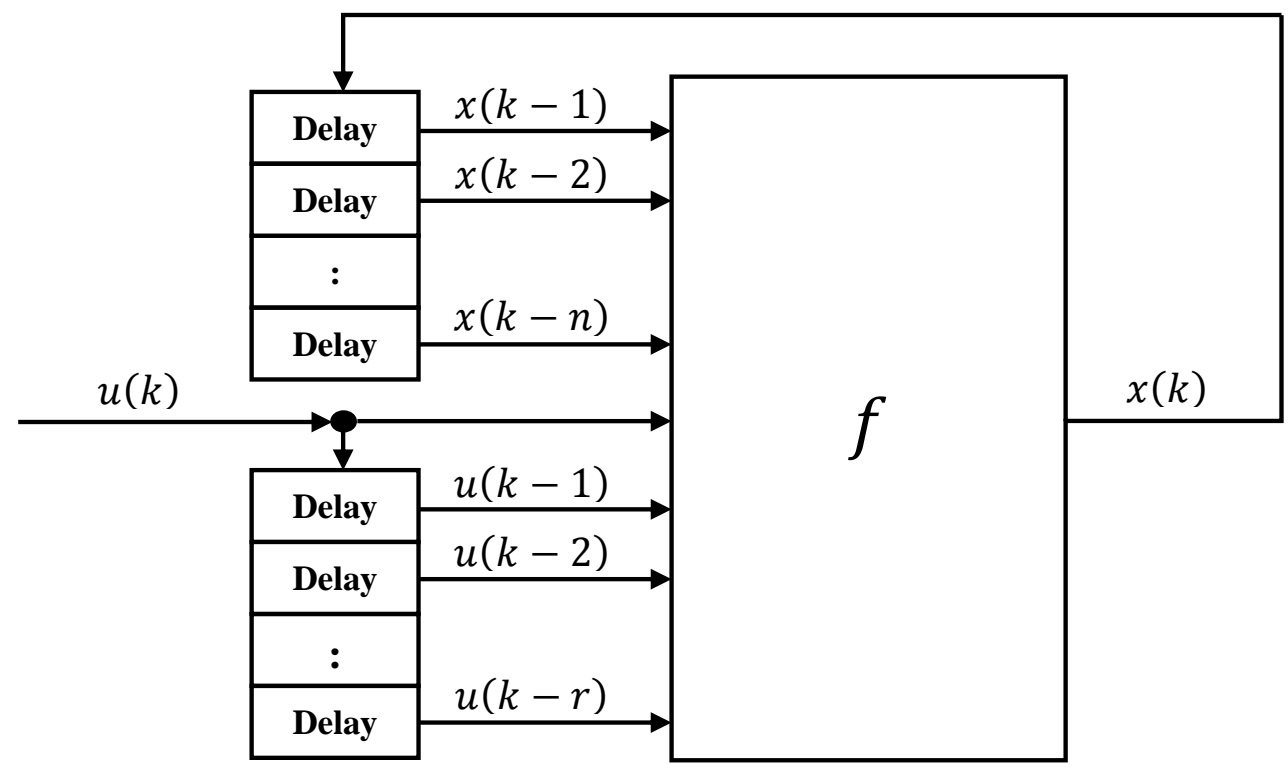

Figure 1. Block diagram of a dynamic system with external inputs based on a static function $f$ and delays

\section{General Framework for FRDS with Smooth FRC}

Let $\underline{v}=[x(k-1), \ldots, x(k-n), u(k-1), \ldots, u(k-r)]$. It is the vector of all inputs to the function block $\mathrm{f}$ and is of length $\eta:=n+r$. The function block of Fig. 1 is shown as the outer box with broken line in Fig. 2. In this figure the three main parts of an FRM is depicted, the Fuzzifier, the fuzzy relational matrix along with the fuzzy relational composition, and the Defuzzifier.

We consider the part types as follows:

- Fuzzifier: Standard Fuzzifier which is a fuzzifier with triangular membership functions with sum-normality property and with its centers equally spaced in the normalized nonfuzzy space.

- Fuzzy relational matrix: An $(\eta+1)$-dimensional matrix (instead of 2-dimensional matrix) with its elements from $[0,1]$. We denote this matrix with both $R_{\eta+1}$ and $R$.

- Fuzzy relational composition: This will be defined exactly in this section. It is of type s-t which composes the relational matrix $R$ with the fuzzy vectors $\underline{a}_{1}, \ldots, \underline{a}_{\eta}$ as we define in the sequel.

- Defuzzifier: Weighted average defuzzifier which is defined as $y=\sum_{j=1}^{q} b_{j} c_{j} / \sum_{j=1}^{q} b_{j}$, where $b_{j}$ 's are the elements of $\underline{b}$, and $c_{j}$ 's are the centers of the membership functions of the 
defuzzifier, and $q$ is the number of linguistic terms of the defuzzifier and hence the length of the vectors $\underline{b}$ and $\underline{c}$.

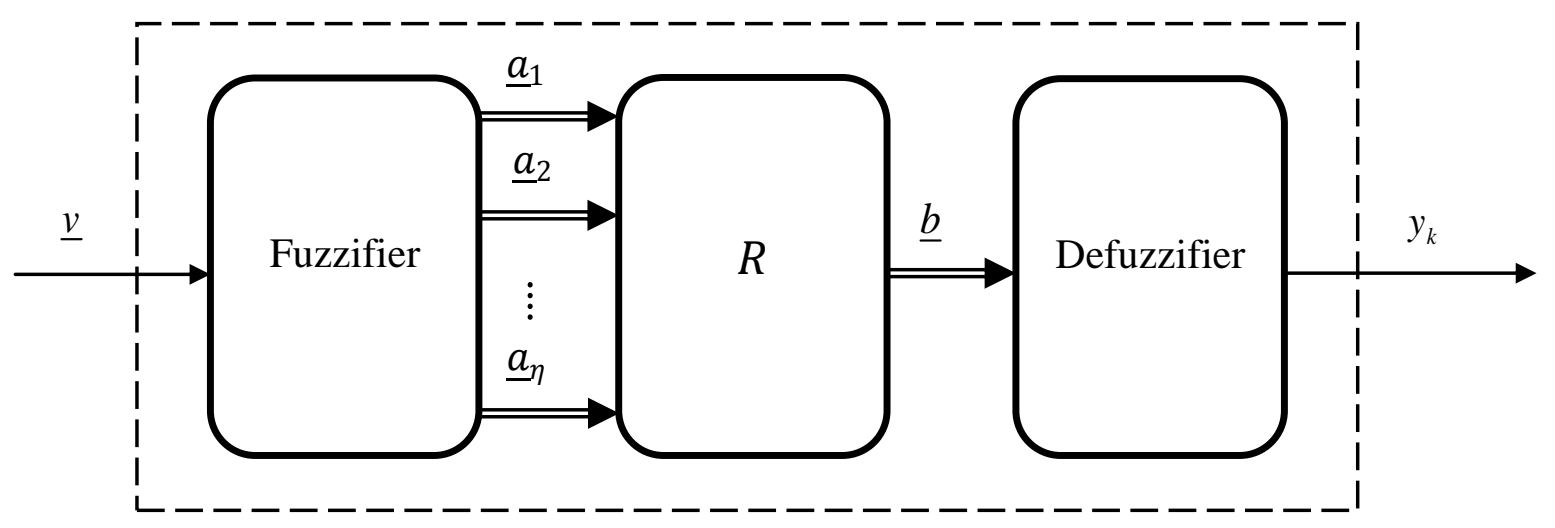

Figure 2. Main parts of an FRM

Note 1: For any scalar $\alpha \in[0,1]$ and any $n$-dimensional relational matrices $R_{n}^{1}=\left[r_{n}^{1}\left(i_{1}, \ldots, i_{n}\right)\right], \ldots$, $R_{n}^{q}=\left[r_{n}^{q}\left(i_{1}, \ldots, i_{n}\right)\right]$ where $r_{n}^{j}\left(i_{1}, \ldots, i_{n}\right) \in[0,1]$, we define the following bundle t-norm and t-conorm operations.

$$
\begin{aligned}
& t\left(\alpha, R_{n}^{j}\right):=\left[t\left(\alpha, r_{n}^{j}\left(i_{1}, \ldots, i_{n}\right)\right)\right] \\
& S\left(R_{n}^{j}, R_{n}^{k}\right):=\left[S\left(r_{n}^{j}\left(i_{1}, \ldots, i_{n}\right), r_{n}^{k}\left(i_{1}, \ldots, i_{n}\right)\right)\right]
\end{aligned}
$$

Furthermore due to the associativity of the t-conorm, the t-conorm on more than two matrices can be defined as follows.

$$
S_{i=1}^{q}\left(R_{n}^{i}\right):=\left[S_{i=1}^{q} r_{n}^{i}\left(i_{1}, \ldots, i_{n}\right)\right]
$$

Also note the difference between $S$ and $s$ in this text; $s_{i=1}^{q}($.) operates on some scalars while $S_{i=1}^{q}($. )operates on matrices and performs as a kind of bundle t-conorm.

Now let us define the way $\underline{b}$ is calculated effectively from $\underline{a}_{1}, \ldots, \underline{a}_{\eta}$ under $R$, and let us denote the $(\eta+1)$-dimensional matrix $R$ with $R_{\eta+1}$. The fuzzifier is assumed the same for all the elements of $\underline{v}$, and hence we denote by $q$ the number of linguistic terms of the fuzzifiers and the defuzzifier, $q_{1}=\cdots=q_{\eta}=q$. First note that for every $j \in\{1, \ldots, \eta\}$ we have $\underline{a}_{j}=\left[\underline{a}_{j}(1), \ldots, \underline{a}_{j}(q)\right], \underline{b}_{j}=$ $\left[\underline{b}_{j}(1), \ldots, \underline{b}_{j}(q)\right]$ where $\underline{a}_{j}(i), \underline{b}_{j}(i) \in[0,1]$. The structure of the $\underbrace{q \times \cdots \times q}_{(\eta+1)}$ relational matrix $R_{\eta+1}=\left[r_{\eta+1}\left(i_{1}, \ldots, i_{\eta+1}\right)\right]$ is determined by an identification process which we will present in the next section.

The operation of $R$ on the fuzzy vectors $\underline{a}_{1}, \ldots, \underline{a}_{\eta}$ is denoted by $\underline{b}=\underline{a}_{\eta} \circ \cdots \circ \underline{a}_{1} \circ R_{\eta+1}$. We define this operation as follows:

$$
\begin{aligned}
& \Lambda_{j+1}:=\underline{a}_{\eta+1-j} \widehat{\otimes} R_{j+1}, \forall j \in\{\eta, \ldots, 1\} \\
& R_{j}=S_{i=1}^{q}\left(\Lambda_{j+1}\right), \forall j \in\{\eta, \ldots, 1\} \\
& \underline{b}=R_{1}
\end{aligned}
$$

where the operators $S_{i=1}^{q}($.$) and \widehat{\otimes}$ are defined for the $j$-dimensional relational matrix $R_{j}$ using bundle operations of Note 1 , as follows. Note that in this notation, $\Lambda_{j}$ and $R_{j}$ are $j$-dimensional matrices.

$$
\begin{aligned}
& S_{i=1}^{q}\left(R_{j}\right):=S_{i=1}^{q}\left[r_{j}\left(i_{1}, \ldots, i_{j-1}, i\right)\right], \forall i_{1}, \ldots, i_{j-1} \in\{1, \ldots, q\} \\
& \underline{a} \widetilde{\otimes} R_{j}:=\left[t\left(\underline{a}(i),\left[r_{j}\left(i_{1}, \ldots, i_{j-1}, i\right)\right]\right)\right]
\end{aligned}
$$

This completes the modeling framework for our FRDS. In the next step, the modeling framework (1) should be identified to represent an actual dynamic system. The identification algorithm is presented in the next section. Note that using a smooth fuzzy relational composition with the FRDS framework yields the smooth FRDS. 


\section{Identification}

In this section an actual dynamic system with known inputs and outputs is assumed and a smooth FRDS is to be identified to represent that system as good as possible. For simplicity let us denote the derivatives of the t-norm $t$ and the t-conorm $s$ as follows.

$$
\begin{aligned}
& \dot{T}(a, b):=\left.\frac{\partial t(x, y)}{\partial x}\right|_{(x, y)=(a, b)} \\
& \dot{S}(a, b):=\left.\frac{\partial s(x, y)}{\partial x}\right|_{(x, y)=(a, b)}
\end{aligned}
$$

The approach of back propagation is used to identify the smooth FRDS iteratively. Sensitivity of the squared error of the output to the relational matrix parameters is used in this regard to tune the parameters as follows.

$$
\begin{aligned}
& e:=y-y^{t}, E:=e^{2} \\
& \left\{\begin{array}{c}
\Delta r_{\left(l, k_{1}, \ldots, k_{\eta}\right)}=-\mu \frac{\partial E}{\partial r_{\left(l, k_{1}, \ldots, k_{\eta}\right)}} \\
r_{\left(l, k_{1}, \ldots, k_{\eta}\right)}(k+1)=r_{\left(l, k_{1}, \ldots, k_{\eta}\right)}(k)+\Delta r_{\left(l, k_{1}, \ldots, k_{\eta}\right)}
\end{array}, \quad \forall l, k_{1}, \ldots, k_{\eta} \in\{1, \ldots, q\}\right.
\end{aligned}
$$

where $y^{t}$ is the target output, i.e., the output of the actual system, $k$ is the time instant, and $\mu$ is the tuning rate which can be adjusted dynamically through the process of tuning the parameters. Therefore for fixed $l, k_{1}, \ldots, k_{\eta}$ the sensitivity can be calculated as follows.

$$
\begin{aligned}
& \frac{\partial E}{\partial r_{\left(l, k_{1}, \ldots, k_{\eta}\right)}}=\frac{\partial y}{\partial r_{\left(l, k_{1}, \ldots, k_{\eta}\right)}} \frac{\partial e}{\partial y} \frac{\partial E}{\partial e}=\left(\sum_{j=1}^{q} \frac{\partial \underline{b}(j)}{\partial r_{\left(l, k_{1}, \ldots, k_{\eta}\right)}} \frac{\partial y}{\partial \underline{b}(j)}\right)(1)(2 e)=2 e \frac{\partial \underline{b}(l)}{\partial r_{\left(l, k_{1}, \ldots, k_{\eta}\right)}} \frac{\partial y}{\partial \underline{b}(l)} \\
& \frac{\partial y}{\partial \underline{b}(l)}=\frac{c_{l}-y}{\sum_{j=1}^{q} b_{j}} \\
& \frac{\partial \underline{b}(l)}{\partial r_{\left(l, k_{1}, \ldots, k_{\eta}\right)}}=\frac{\partial\left(R_{1}(l)\right)}{\partial\left(R_{\eta+1}\left(l, k_{1}, \ldots, k_{\eta}\right)\right)}=\frac{\partial\left(\Lambda_{\eta+1}\left(l, k_{1}, \ldots, k_{\eta}\right)\right)}{\partial\left(R_{\eta+1}\left(l, k_{1}, \ldots, k_{\eta}\right)\right)} \underbrace{\frac{\partial\left(R_{\eta}\left(l, k_{1}, \ldots, k_{\eta-1}\right)\right)}{\partial\left(\Lambda_{\eta+1}\left(l, k_{1}, \ldots, k_{\eta}\right)\right)}}_{\alpha_{\eta+1}} \cdots \underbrace{\ldots}_{\beta_{\eta+1}} \underbrace{\frac{\partial\left(\Lambda_{2}\left(l, k_{1}\right)\right)}{\partial\left(R_{2}\left(l, k_{1}\right)\right)}}_{\alpha_{2}} \underbrace{\frac{\partial\left(R_{1}(l)\right)}{\partial\left(\Lambda_{2}\left(l, k_{1}\right)\right)}}_{\beta_{2}} \\
& \alpha_{i}:=\frac{\partial\left(\Lambda_{i}\left(l, k_{1}, \ldots, k_{i-1}\right)\right)}{\partial\left(R_{i}\left(l, k_{1}, \ldots, k_{i-1}\right)\right)}, \forall i \in\{2, \ldots, \eta+1\} \\
& \beta_{i}:=\frac{\partial\left(R_{i-1}\left(l, k_{1}, \ldots, k_{i-2}\right)\right)}{\partial\left(\Lambda_{i}\left(l, k_{1}, \ldots, k_{i-1}\right)\right)} \\
& \frac{\partial \underline{b}(l)}{\partial r_{\left(l, k_{1}, \ldots, k_{\eta}\right)}}=\prod_{i=2}^{n+1} \alpha_{i} \beta_{i}
\end{aligned}
$$

The expansion is finally wrapped up as follows.

$$
\begin{aligned}
& \alpha_{i}:=\dot{T}\left(R_{i}\left(l, k_{1}, \ldots, k_{i-1}\right), \underline{a}_{\eta+2-i}\left(k_{i-1}\right)\right), \forall i \in\{2, \ldots, \eta+1\} \\
& \beta_{i}:=\dot{S}\left(\Lambda_{i}\left(l, k_{1}, \ldots, k_{i-1}\right), s_{\substack{j=1 \\
j \neq k_{i-1}}} \Lambda_{\eta+1}\left(l, k_{1}, \ldots, k_{\eta-1}, j\right)\right)
\end{aligned}
$$

Therefore the tuning procedure is proposed as follows.

$$
\begin{aligned}
& r_{\left(l, i_{1}, \ldots, i_{\eta}\right)}(k+1)=r_{\left(l, i_{1}, \ldots, i_{\eta}\right)}(k)+\Delta r_{\left(l, i_{1}, \ldots, i_{\eta}\right)} \\
& \Delta r_{\left(l, i_{1}, \ldots, i_{\eta}\right)}=-2 \mu e \frac{c_{l}-y}{\sum_{j=1}^{q} b_{j}} \prod_{i_{n}=2}^{\eta+1} \alpha_{i_{n}} \beta_{i_{n}}
\end{aligned}
$$

\section{Simulation Results}

In this section some benchmark systems as well as some actual systems are modeled using the smooth FRDS driven in the previous sections.

\subsection{Box-Jenkins gas furnace [6]}

This data set contains 296 input/output pair and is one of the most popular benchmark problems. The mean square error of the approximations is calculated in the literature to assess the performence. Table 1 shows some of these results that we could find. The simulation result is depicted in Fig. 3. 

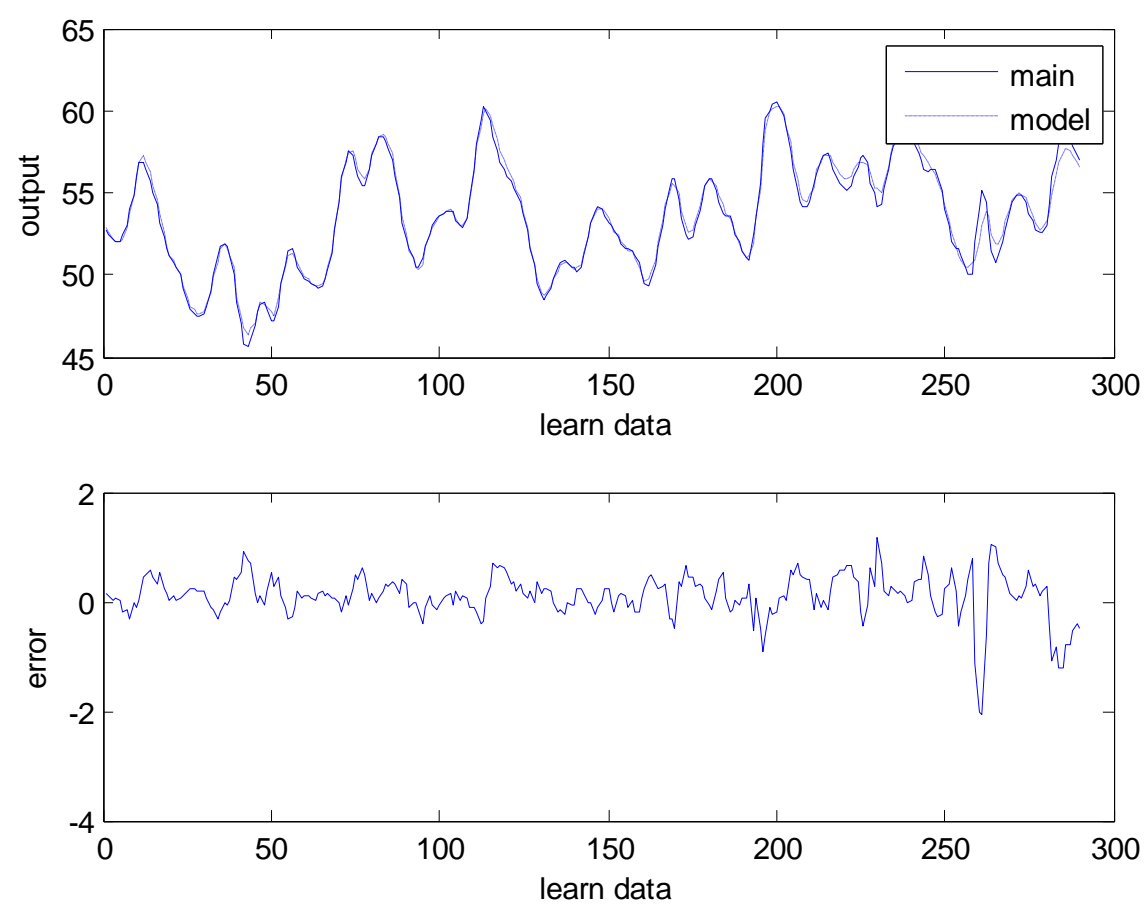

Figure 3. Actual output, model output, and error curve for modeling the Box-Jenkins gas furnace with smooth FRDS

We achieved mean squared error $J=0.18$ using the proposed smooth FRDS framework with pi fuzzy relational composition which is composed of pi t-norm and pi t-conorm as follows. Here $p=2$.

$$
\begin{aligned}
& t(a, b)=1-\sqrt[2]{(1-a)^{2}+(1-b)^{2}-(1-a)^{2}(1-b)^{2}} \\
& s(a, b)=\sqrt[2]{a^{2}+b^{2}-a^{2} b^{2}}
\end{aligned}
$$

data

Table 1. The mean squared error of the outputs of various models for Box-Jenkins gas furnace

\begin{tabular}{|c|c|}
\hline Paper & $J$ \\
\hline$[6]$ & 0.710 \\
\hline$[2]$ & 0.478 \\
\hline$[7]$ & 0.469 \\
\hline$[8]$ & 0.407 \\
\hline$[9]$ & 0.328 \\
\hline$[10]$ & 0.312 \\
\hline
\end{tabular}

In the next step, only the first half of the data is used for identification and the second of half of the data is used for evaluation of the model. This lead to mean square error $J_{\text {learn }}=0.0672$ for the identification part and $J_{\text {test }}=0.3888$ for the evaluation part. The result is depicted in Fig. 4 . 

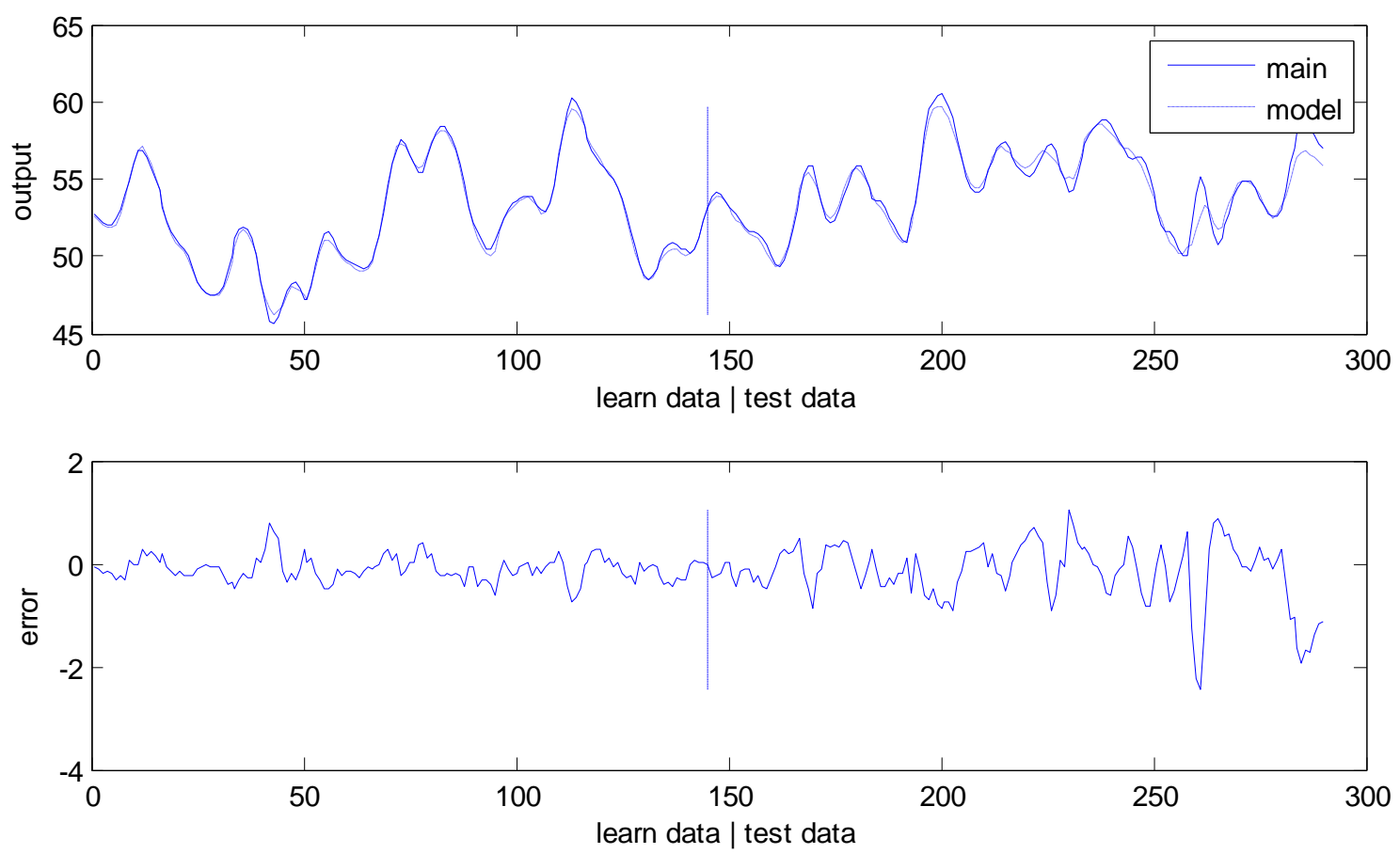

Figure 4. Actual output, model output, and error curve for modeling the Box-Jenkins gas furnace with smooth FRDS

\subsection{Electromechanical system}

This system consist of an inverted pendulum coupled to a separately excited DC motor (SEDCM) via a gear box. The input is the armature voltage and the output is the vertical angle of the pendulum. The system is in open-loop and no controller is involved. So, several experiments have been performed to produce the required data. The output datais shown in Fig. 5 together with the output of the model. A part of this figure is magnified in Fig. 6 . The overall mean square error is $J=0.0023$.

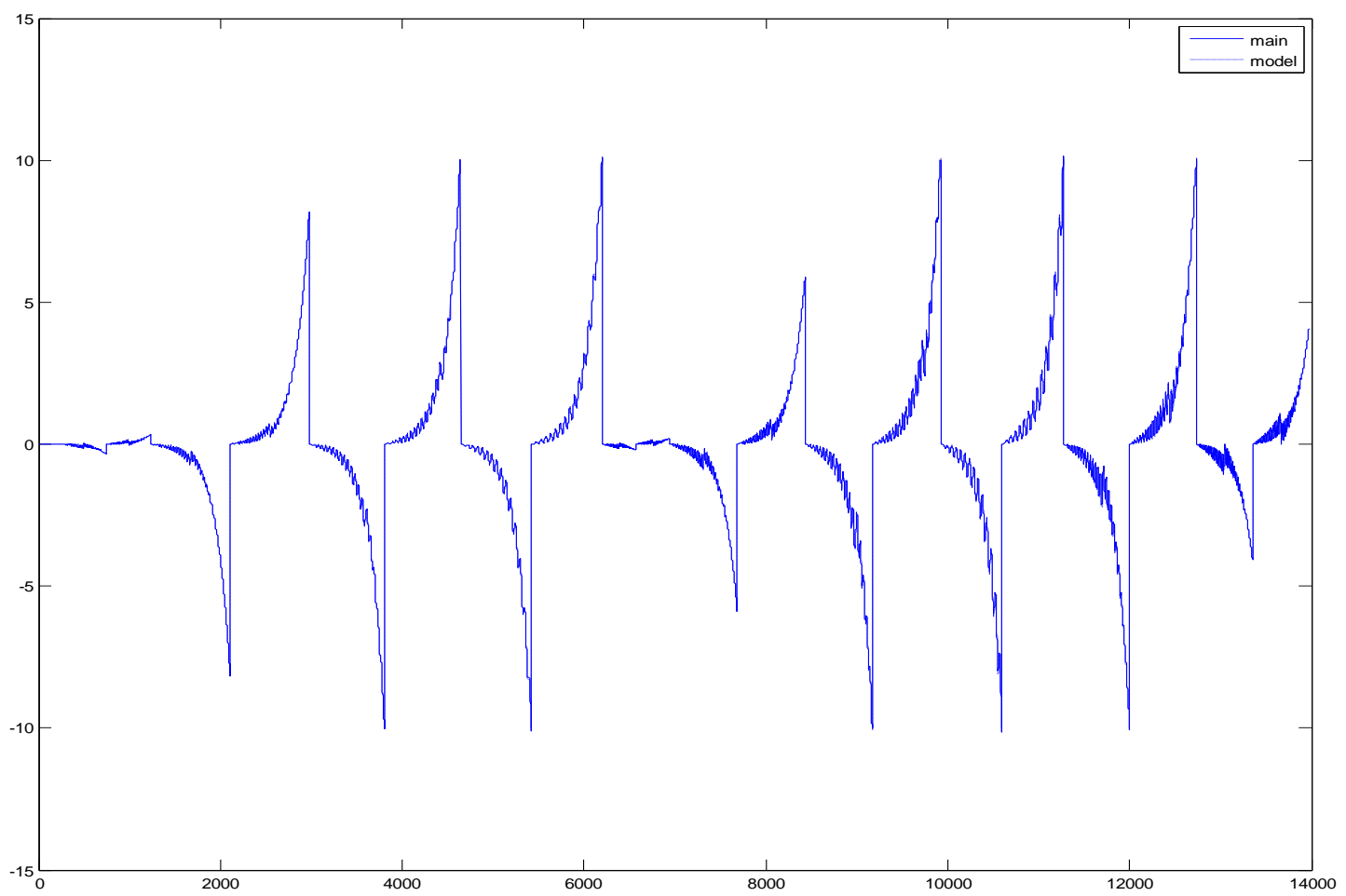

Figure 5. Actual output, model output, and error curve for modeling the electromechanical system with smooth FRDS 


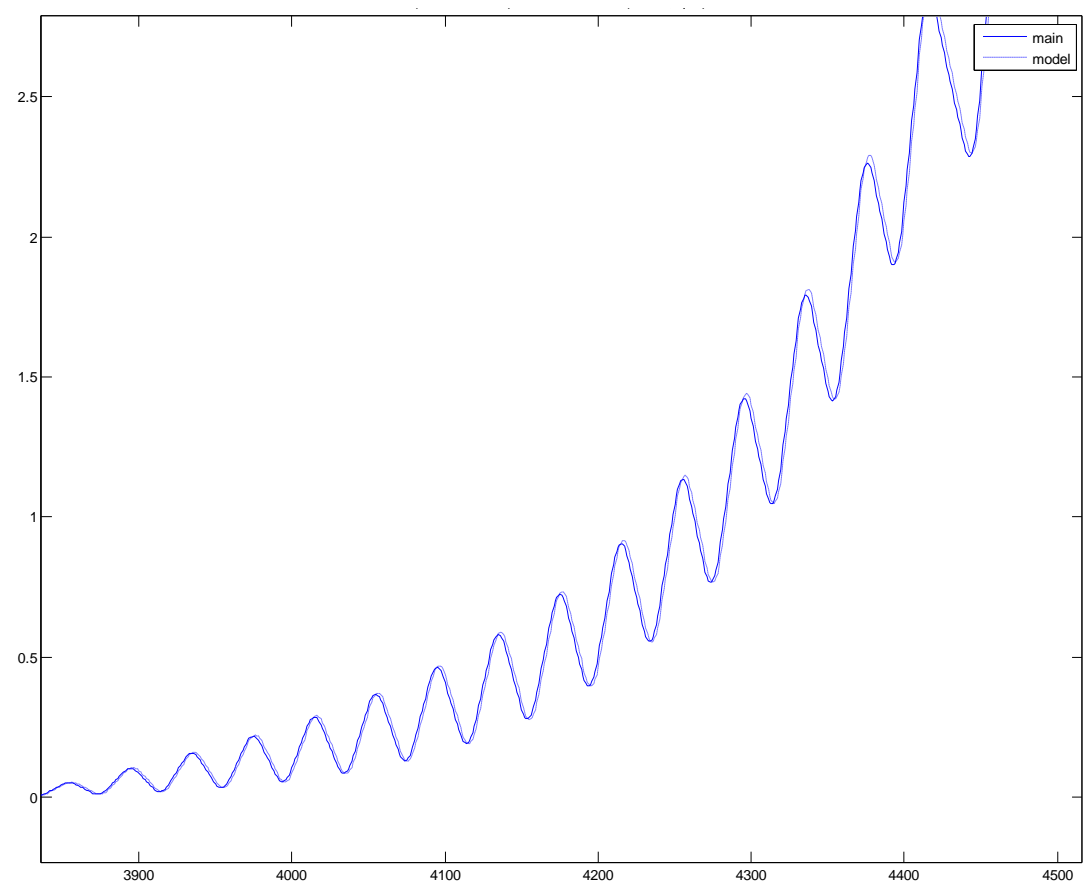

Figure 6. Magnification of a part of Fig. 5

\subsection{Nonlinear dynamical system}

The system is described by the following equations.

$$
\left\{\begin{array}{l}
x_{1}=y(k) \\
x_{2}=y(k-1) \\
x_{3}=y(k-2), y(k+1)=\frac{x_{1} x_{2} x_{3} x_{5}\left(x_{3}-1\right)+x_{4}}{1+x_{2}^{2}+x_{3}^{2}} \\
x_{4}=u(k) \\
x_{5}=u(k-1)
\end{array}\right.
$$

where the input is as follows.

$$
u(k)=\left\{\begin{array}{cc}
\sin (\pi k / 25) & 0 \leq k \leq 250 \\
1 & 250 \leq k \leq 500 \\
-1 & 500 \leq k \leq 750 \\
0.3 \sin (\pi k / 25)+0.1 \sin (\pi k / 32)+0.6 \sin (\pi k / 10) & 750 \leq k \leq 1000
\end{array}\right.
$$

To model the system, 1000 data pairs were generated by Simulink. The first 200 data is used for identification and the next 800 data is used for evaluation which yields $J_{\text {learn }}=0.0191$ for the identification part and $J_{\text {test }}=0.0177$ for the evaluation part. The result can be seen in Fig. 7 . 

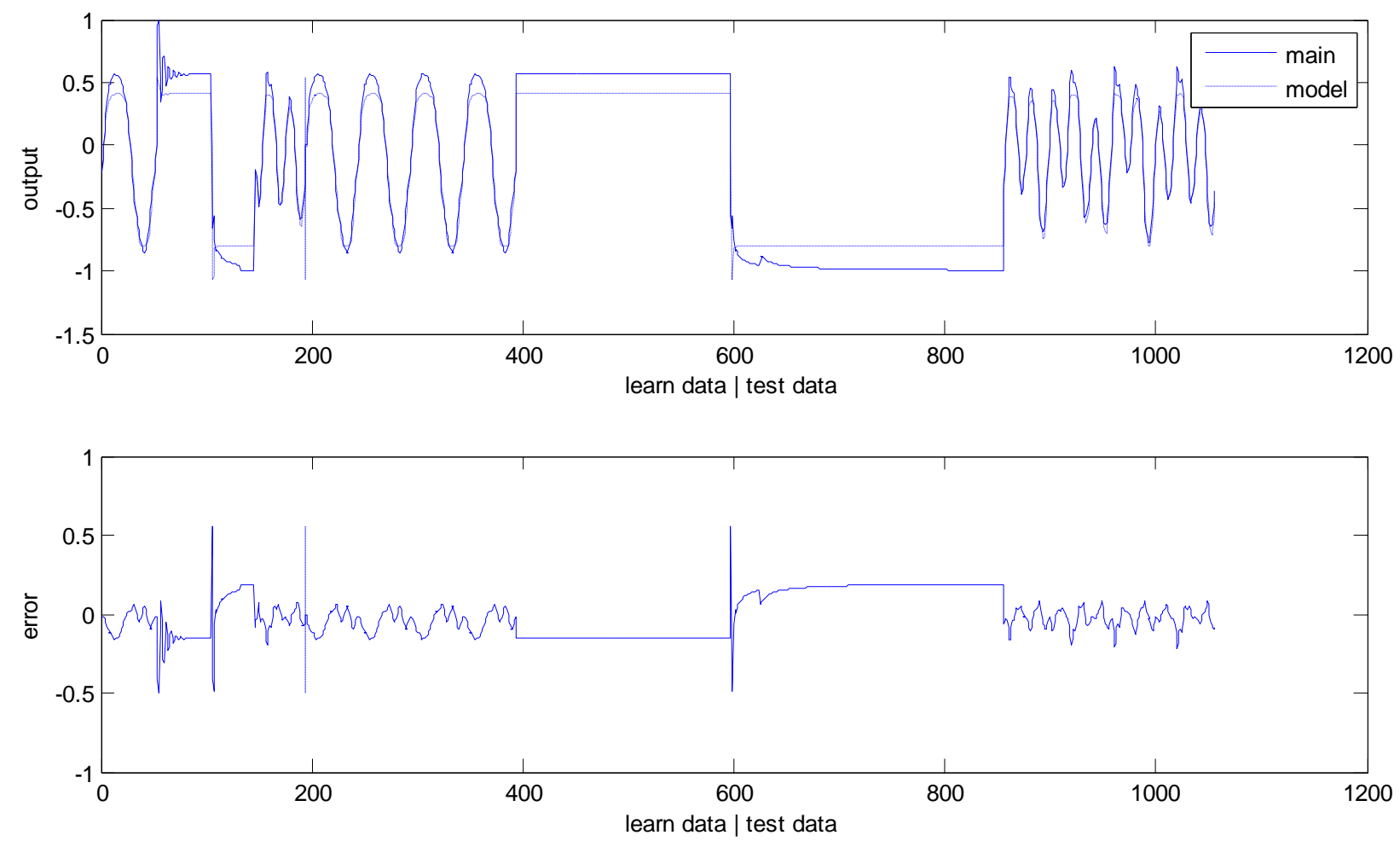

Figure 7. Actual output, model output, and error curve for modeling the nonlinear system with smooth FRDS

\section{Conclusion}

In this paper a general framework for fuzzy linguistic modeling of dynamical systems was proposed based on fuzzy relational models and smooth fuzzy compositions which we call it smooth FRDS. To this end, a modeling framework and an identification algorithm was introduced. Simulation results proved the modeling capability of the proposed smooth FRDS.

\section{References}

[1] E. Sanchez, "Resolution of Composite Fuzzy Relation Equations", Information and Control, Vol. 30, No. 1, pp. 38-48, 1976.

[2] W. Pedrycz, "An identification algorithm in fuzzy relational systems", Fuzzy Sets and Systems, Vol. 13, pp. 153167, 1984.

[3] W. Pedrycz, "Fuzzy neural networks and neurocomputations", Fuzzy Sets and Systems, Vol. 56, pp. 1-28, 1993. [4] J. N. Ridley, I. S. Shaw, and J. J. Kruger, "Probabilistic fuzzy model for dynamic systems", Electronics Letters, Vol. 24, pp. 890-892, 1988.

[5] A. Aghili Ashtiani, and M. B. Menhaj, "Numerical Solution of Fuzzy Relational Equations Based on Smooth Fuzzy Norms", Soft Computing,Vol. 14, No. 6, pp. 545-557, 2010; DOI: 10.1007/s00500-009-0425-1.

[6] V. M. Box, G. M. Jenkins, "Time Series Analysis", Forecasting and Control, HoldenDay, San Francisco, CA, 1970.

[7] R. M. Tong, "The evolution of fuzzy models derived from experimental data", Fuzzy Sets and Systems, Vol. 4, pp. 1-12, 1980.

[8] Y. C. Lee, C. Hwang, and Y. P. Shih, "A Combined Approach to Fuzzy Model Identification", IEEE Transactions on Systems, Man, and Cybernetics, Vol. 24, No 5, may 1994.

[9] C.W. Xu, and Y. Z. Lu, "Fuzzy model identification and self-learning for dynamic systems", IEEE Transactions on System, Man, and Cybernetics, Vol. SMC-17, No. 4, July/August, 1987.

[10] P. J. Costa Branco, J. A. Dente, "A New Algorithm for On-Line Relational Identification of Nonlinear Dynamic Systems", Instituto Superior Tknico (I.S.T.). 